\title{
Critical currents and giant non-dissipative drag for superfluid electron-hole pairs in quantum Hall multilayers
}

\author{
L Yu Kravchenko, D V Fil \\ Institute for Single Crystals, National Academy of Science of Ukraine, Lenin av. 60, \\ Kharkov 61001, Ukraine \\ E-mail: sotice@mail.ru, fil@isc.kharkov.ua
}

\begin{abstract}
Superfluid properties of electron-hole pairs in a quantum Hall four-layer system are investigated. The system is considered as a solid state realization of a two-component superfluid Bose gas with dipole-dipole interaction. One superfluid component is formed in the top bilayer and the other component - in the bottom one. We obtain the dispersion equation for the collective mode spectrum and compute the critical parameters (the critical interlayer distance and the critical currents) versus the filling factor. We find that the critical currents of the components depend on each other. The maximum critical current of a given component can be reached if the current of the other component is equal to zero. The non-dissipative drag effect between the components is studied. It is shown that in the system considered the drag factor is very large. Under appropriate conditions it can be about 10 per sent, that is at least in three order larder than one predicted for two-component atomic Bose gases.
\end{abstract}

PACS numbers: 03.75.Kk, 03.75.Mn, 73.43.Lp 


\section{Introduction}

Among the objects that demonstrate Bose-Einstein condensation or superconductivity considerable attention is given to two-component systems. Particularly, beginning from the paper by Andreev and Bashkin [1] the possibility of a non-dissipative drag between superfluid (superconducting) components moving with different velocities was discussed [2, 3, 4, 5, 6] (see also the review [7]). The Andreev-Bashkin effect was also considered in astrophysics in the context of superfluid models of neutron stars [8, 9]. A related problem - the critical velocities in two-component superfluid systems was studied in a recent paper [10]. It was shown that critical velocities are essentially different in the case when two components move with the same velocities and in the case when one of the components does not move. In the latter case the critical velocity of a moving component can be much higher.

Although two-component superfluid atomic Bose gases have been realized in laboratories [11], there are certain problems in experimental observation of the effects caused by relative motion of the components. On the one hand, it is not so simple to create a relative flow of superfluid components in a mixture of Bose gases. On the other hand, a spatial separation of components takes place in two-component mixtures confined in a trap. In the gases with point interaction the spatial separation results in a disappearance of the effects caused by inter-specie interaction. To overcome these difficulties one can turn to systems where components can be kept spatially separated in a controllable way (that gives a possibility to provide a flow of the components with different velocities). The interaction between the components in such systems should contain a long-range part. For example, one can deal with a Bose gas with dipole-dipole interaction confined in a double-layer trap [5].

In this paper we study a solid-state system where a two-component Bose gas with the dipole-dipole interaction can be realized. We consider a multilayer electron system where electrons from one layer couple with holes from the adjacent layer. Since such Bose particles (electron-hole pairs) have a small mass they may demonstrate superfluid behavior at rather high temperatures (much higher than required for the Bose-Einstein condensation of alkali metal vapors). Electron-hole pairs in such systems have large dipole momentum and the dipole-dipole interaction determines, in the main part, collective properties of a gas of such pairs.

To be more specific, we consider a four-layer electron system in a strong perpendicular to the layers magnetic field (a multilayer quantum Hall system). For bilayer quantum Hall systems with total filling factor equal to unity the theory predicts [12] the existence of a superfluid condensate of indirect excitons in the systems. An indirect exciton corresponds to a bound state of an electron belonging to one layer and a hole (an empty state in the lowest Landau level) belonging to the other layer. This prediction was partially confirmed in experiments [13, 14, 15]. Bose-Einstein condensation of metastable (optically generated) indirect excitons in zero magnetic field was also observed [16, 17]. As was shown in [18], multi-component excitonic superfluid 
condensates can be realized in multilayer quantum Hall systems with even number of the layers and the average filling factor per layer equal to one-half. According to [18], electron-hole pairs emerge in separate bilayers, i.e., a given excitonic component belongs to a given double-layer complex. Here we consider a four-layer system with the filling factors of the layers $\nu_{1}=\nu_{\mathrm{T}}, \nu_{2}=1-\nu_{\mathrm{T}}, \nu_{3}=\nu_{\mathrm{B}}, \nu_{4}=1-\nu_{\mathrm{B}}$. In such a system one specie is formed by coupled electrons and holes from the layers 1 and 2, while the other specie - by coupled carriers from the layers 3 and 4 (Figure 1).

In the system considered a flow of electron-hole pairs is equivalent to two oppositely directed electrical currents in the adjacent layers. Therefore, a superfluid state of such pairs can be considered as a specific superconducting state. Counterflow supercurrents can carry an electrical current from the source situated at one end of the system to the load situated at the opposite end (if the interlayer tunnelling is negligible small, the dissipation is negligible, as well [19]). Experimentally one can provide separate contacts to each layer. It allows to control and measure the supercurrents in each bilayer complex. Therefore, it is more appropriate to formulate the problems not in terms of superfluid velocities, but in terms of supercurrents.

In this paper we address two problems. First, we investigate critical currents in a four-layer quantum Hall system. We show that the behavior of critical currents is qualitative the same as of critical velocities in two-component Bose gases (with the important advantage that the effect in multilayers can be registered by electrical measurements). Then, we consider the non-dissipative drag between the components and compute the drag factor. We predict very large drag factor for quantum Hall multilayers: under appropriate conditions it can reach 10 per sent (in three order higher than the most optimistic estimates for atomic Bose gases).

Our study is based on the analysis of collective mode spectra. We follow the approach proposed in [20] for the study of bilayer systems with zero imbalance of filling factors $\left(\nu_{1}=\nu_{2}=1 / 2\right)$. In Sec.2 we extend the approach [20] for the case of an arbitrary imbalance. In Sec 3 we obtain the spectra of excitations for the four-layer system, find the critical interlayer distance versus the filling factor, and obtain the relation between the critical currents. The non-dissipative drag effect is considered in Sec4,

\section{The approach}

Let us begin with the discussion of a mechanism that determines critical supercurrents in quantum Hall bilayers (multilayers). In bulk superconductors the restriction on the value of the supercurrent emerges from the requirement that the magnetic field produced by electrical currents should be lower than the thermodynamic critical magnetic field. For thin films the critical magnetic field is higher than for bulk superconductors, and at small thickness $w$ of the film it increases by the law $H_{\mathrm{c}} \propto 1 / w$. Due to almost two-dimensional character of conducting layers in quantum Hall systems the critical magnetic field should be high. The critical current is determined by an essentially different mechanism. This mechanism is just the generalization of the Landau mechanism of destruction of 
superfluidity (governed by the Landau criterion). The critical current can be found from the requirement that in a superconducting state the energies of all collective excitations being real valued and positive. Going ahead we note that electrical currents close to critical ones produce magnetic fields much smaller than the terrestrial magnetic field. And, indeed, the magnetic mechanism of destruction of superconductivity is irrelevant for the quantum Hall bilayers (multilayers).

For more transparency, we will describe the approach with reference to a bilayer system. Let a double-layer electron system is situated in a perpendicular to the layers magnetic field $B$. The electron density $\rho=\rho_{1}+\rho_{2}$ satisfies the condition $\nu_{\text {tot }}=2 \pi l^{2} \rho=1$, where $l=\sqrt{\hbar c / e B}$ is the magnetic length, i.e. the filling factor of the layer 1 is $\nu_{1}=\nu(\nu<1)$, and the filling factor of the layer 2 is $\nu_{2}=1-\nu$. The value $\nu$ is the parameter of the problem. Since $\nu_{1}, \nu_{2}<1$ all the carriers belong to the lowest Landau level (under assumption that the Coulomb energy is small compared to the energy gap between the Landau levels). We take the Hamiltonian in the lowest Landau level approximation:

$$
H=\frac{1}{2 S} \sum_{n, n^{\prime}=1}^{2} \sum_{\mathbf{q}} V_{n, n^{\prime}}(q)\left\{\rho_{n}(\mathbf{q}) \rho_{n^{\prime}}(-\mathbf{q})-\delta_{n, n^{\prime}} \rho_{n}(0) \exp \left(-\frac{l^{2} q^{2}}{2}\right)\right\}
$$

where $S=L_{\mathrm{x}} L_{\mathrm{y}}$ is the area of the layer, $V_{n, n^{\prime}}(q)=2 \pi e^{2} \mathrm{e}^{-d q\left|n-n^{\prime}\right|} / \varepsilon q$ is the Fouriercomponent of the Coulomb potential, $d$ is the distance between the layers, $\varepsilon$ is the dielectric constant, and

$$
\rho_{n}(q)=\sum_{X} c_{n}^{+}\left(X+\frac{q_{\mathrm{y}} l^{2}}{2}\right) c_{n}\left(X-\frac{q_{\mathrm{y}} l^{2}}{2}\right) \exp \left(\mathrm{i} q_{\mathrm{x}} X-\frac{q^{2} l^{2}}{4}\right)
$$

is the Fourier-component of the electron density in the $n$-th layer. In (2) $c_{n}^{+}(X)$ and $c_{n}(X)$ are the creation and annihilation operators for electrons in the $n$-th layer in the state described by the wave function $\psi_{X}(\mathbf{r})=\exp \left(-\mathrm{i} X y / l^{2}-(x-X)^{2} / 2 l^{2}\right)$. We imply that the interlayer tunnelling amplitude $t$ is much smaller than the Coulomb energy $E_{c}=e^{2} / \varepsilon l$ and neglect the tunnelling in the Hamiltonian (11). We will discuss the validity of such an approximation in more details at the end of this section. Here we just mention that in the bilayer systems used in experiments the tunneling amplitude $t \approx 50 \mu \mathrm{K}[13$, that is in 6 order smaller than the Coulomb energy.

The state with electron-hole pairing can be described by a BCS-like many-body wave function $|\Psi\rangle=\prod_{X}\left[u_{X}+v_{X} h_{1}^{+}(X) c_{2}^{+}(X)\right]|v a c\rangle$, where $h_{1}^{+}$is the creation operator of the hole in the layer 1 and $|v a c\rangle$ is the vacuum state defined as a state with completely filled layer 1 and empty layer 2 . The $u-v$ coefficients satisfy the condition $\left|u_{X}\right|^{2}+\left|v_{X}\right|^{2}=1$. This function can be presented in another equivalent form

$$
|\Psi\rangle=\prod_{X}\left[\cos \frac{\theta_{X}}{2} c_{1}^{+}(X)+\mathrm{e}^{\mathrm{i} \varphi_{X}} \sin \frac{\theta_{X}}{2} c_{2}^{+}(X)\right]|0\rangle,
$$

where $\theta_{X}=\theta(X)$ and $\varphi_{X}=\varphi(X)$ are arbitrary functions. The quantity $\theta$ can be connected with the local filling factors $\nu_{X, 1(2)}=\left(1 \pm \cos \theta_{X}\right) / 2$. One can see that $\varphi_{X}$ is the phase of the order parameter $\Delta_{X}=\left\langle\Psi\left|c_{1 X}^{+} c_{2 X}\right| \Psi\right\rangle=\mathrm{e}^{\mathrm{i} \varphi_{X}} \sqrt{\nu_{X}\left(1-\nu_{X}\right)}$ which 
corresponds to the electron-hole pairing. At $\theta=$ const and $\varphi=0$ the function (3) in the coordinate representation coincides with the famous $(1,1,1)$ Halperin wave function (see, for instance, [21]).

In the state (3) the energy of the system reads as

$$
\begin{aligned}
& E=\frac{1}{2 L_{\mathrm{y}}} \sum_{X, X^{\prime}}\left\{\left[H\left(X-X^{\prime}\right)-F_{\mathrm{S}}\left(X-X^{\prime}\right)\right] \cos \theta_{X} \cos \theta_{X^{\prime}}\right. \\
& \left.-F_{\mathrm{D}}\left(X-X^{\prime}\right) \sin \theta_{X} \sin \theta_{X^{\prime}} \cos \left(\varphi_{X}-\varphi_{X^{\prime}}\right)\right\},
\end{aligned}
$$

where the quantities

$$
\begin{aligned}
& H(X)=\frac{e^{2}}{2 \varepsilon} \int_{-\infty}^{\infty} \mathrm{d} q \frac{1-\mathrm{e}^{-|q| d}}{|q|} \mathrm{e}^{\mathrm{i} q X-\frac{q^{2} l^{2}}{2}} \\
& F_{\mathrm{S}}(X)=\frac{e^{2}}{2 \varepsilon} \mathrm{e}^{-\frac{X^{2}}{2 l^{2}}} \int_{-\infty}^{\infty} \frac{\mathrm{d} q}{\sqrt{q^{2}+X^{2} / l^{4}}} \mathrm{e}^{-\frac{q^{2} l^{2}}{2}} \\
& F_{\mathrm{D}}(X)=\frac{e^{2}}{2 \varepsilon} \mathrm{e}^{-\frac{X^{2}}{2 l^{2}}} \int_{-\infty}^{\infty} \frac{\mathrm{d} q}{\sqrt{q^{2}+X^{2} / l^{4}}} \mathrm{e}^{-|q| d-\frac{q^{2} l^{2}}{2}}
\end{aligned}
$$

describe the direct Coulomb interaction, the exchange interaction in a given layer, and the exchange interaction between the layers, respectively.

We consider excitations above a homogeneous state with a stationary superflow of electron-hole pairs along the $x$ direction. Such a state corresponds to $\theta_{X}=\theta_{0}$ independent of $X$ and the phase $\varphi_{X}=Q X$ linear in $X$. The energy of the homogeneous state is found from Eq.(4) and reads as

$$
E^{(0)}=\frac{S}{4 \pi l^{2}}\left(\left[\mathcal{H}(0)-\mathcal{F}_{\mathrm{S}}(0)\right] \cos ^{2} \theta_{0}-\mathcal{F}_{\mathrm{D}}(Q) \sin ^{2} \theta_{0}\right) .
$$

Here the functions displayed calligraphically indicate the Fourier-transforms defined as $\mathcal{A}(q)=\left(1 / 2 \pi l^{2}\right) \int_{-\infty}^{\infty} \mathrm{d} X \exp (-\mathrm{i} q X) A(X)$. The explicit expressions for the quantities in (6) are

$$
\begin{aligned}
& \mathcal{H}(q)=\frac{e^{2}}{2 \varepsilon l^{2}} \mathrm{e}^{-\frac{q^{2} l^{2}}{2}} \frac{1-\mathrm{e}^{-d|q|}}{|q|}, \\
& \mathcal{F}_{\mathrm{S}}(q)=\frac{e^{2}}{2 \varepsilon} \int_{0}^{\infty} \mathrm{d} k \mathrm{e}^{-\frac{k^{2} l^{2}}{2}} J_{0}\left(k q l^{2}\right), \\
& \mathcal{F}_{\mathrm{D}}(q)=\frac{e^{2}}{2 \varepsilon} \int_{0}^{\infty} \mathrm{d} k \mathrm{e}^{-\frac{k^{2} l^{2}}{2}} J_{0}\left(k q l^{2}\right) \mathrm{e}^{-k d} .
\end{aligned}
$$

Fluctuations over the stationary state can be parametrized as $\tilde{m}_{z}(X)=\cos \theta_{X}-$ $\cos \theta_{0}$ and $\tilde{\varphi}_{X}=\varphi_{X}-Q X$. The energy of fluctuations in quadratic approximation has the form

$$
E_{\mathrm{fl}}=\frac{S}{4 \pi l^{2}} \sum_{q}\left[\tilde{m}_{z}(-q) \mathcal{K}_{z z}(q) \tilde{m}_{z}(q)+2 \tilde{m}_{z}(-q) \mathcal{K}_{z \varphi}(q) \tilde{\varphi}(q)+\tilde{\varphi}(-q) \mathcal{K}_{\varphi \varphi}(q) \tilde{\varphi}(q)\right] .
$$

In (10) the Fourier-components of the fields $\tilde{m}_{z}(X)$ and $\tilde{\varphi}_{X}$ are defined as

$$
\tilde{m}_{z}(q)=\frac{2 \pi l^{2}}{S} \sum_{X} \tilde{m}_{z}(X) \mathrm{e}^{-\mathrm{i} q X}, \quad \tilde{\varphi}(q)=\frac{2 \pi l^{2}}{S} \sum_{X} \tilde{\varphi}(X) \mathrm{e}^{-\mathrm{i} q X}
$$


In Eq. (10) the components of the matrix $\mathcal{K}$ read as

$$
\begin{gathered}
\mathcal{K}_{z z}(q)=\mathcal{H}(q)-\mathcal{F}_{\mathrm{S}}(q)+\mathcal{F}_{\mathrm{D}}(Q)+\left(\mathcal{F}_{\mathrm{D}}(Q)-\frac{\mathcal{F}_{\mathrm{D}}(q+Q)+\mathcal{F}_{\mathrm{D}}(q-Q)}{2}\right) \cot ^{2} \theta_{0}, \\
\mathcal{K}_{z \varphi}=-\mathrm{i} \cos \theta_{0} \frac{\mathcal{F}_{\mathrm{D}}(q+Q)-\mathcal{F}_{\mathrm{D}}(q-Q)}{2} \\
\mathcal{K}_{\varphi \varphi}(q)=\sin ^{2} \theta_{0}\left[\mathcal{F}_{\mathrm{D}}(Q)-\frac{\mathcal{F}_{\mathrm{D}}(q+Q)+\mathcal{F}_{\mathrm{D}}(q-Q)}{2}\right] .
\end{gathered}
$$

One can note that Eq. (12) diverges at $\theta_{0}=0$ and $\theta_{0}=\pi$ and the approximation (10) violates. But such $\theta_{0}$ correspond to filing factors $\nu_{1}=0$ and $\nu_{2}=1$ (or vice versa). At such filling factor the density of electron-hole pairs is equal to zero and one cannot speak about the spectrum of collective excitation in the gas of the pairs. The cases of $\theta_{0}$ close to 0 or $\pi$ corresponds to low density of the pairs and the approximation (10) is valid under condition that the density fluctuation are small as compared to the ground state density. The latter condition is equivalent to $\left|\tilde{m}_{z}(q)\right| \ll \sin \theta_{0}$.

To quantize the energy (10) one notes that $\tilde{m}_{z}$ and $\tilde{\varphi}$ are the conjugated quantities and the commutator of the operators that correspond to these variables is equal to

$$
\left[\hat{m}_{z}(q), \hat{\varphi}\left(q^{\prime}\right)\right]=-2 \mathrm{i} \frac{2 \pi l^{2}}{S} \delta_{q,-q^{\prime}}
$$

The operators $\hat{m}_{z}(q)$ and $\hat{\varphi}(q)$ can be expressed in terms of Bose creation and annihilation operators in a common way

$$
\hat{m}_{z}(q)=A\left(b_{q}+b_{-q}^{+}\right), \hat{\varphi}(q)=\mathrm{i} B\left(b_{q}-b_{-q}^{+}\right),
$$

where the amplitudes satisfy the condition $A B=2 \pi l^{2} / S$. Replacing the variables $\tilde{m}_{z}(q)$ and $\tilde{\varphi}(q)$ in (10) with the operators (16) and requiring vanishing of the terms containing two creation (two annihilation) operators one finds the explicit expressions for the amplitudes

$$
A=\sqrt{\frac{2 \pi l^{2}}{S}}\left(\frac{\mathcal{K}_{\varphi \varphi}(q)}{\mathcal{K}_{z z}(q)}\right)^{\frac{1}{4}}, \quad B=\sqrt{\frac{2 \pi l^{2}}{S}}\left(\frac{\mathcal{K}_{z z}(q)}{\mathcal{K}_{\varphi \varphi}(q)}\right)^{\frac{1}{4}} .
$$

As a result one obtains the Hamiltonian for the collective excitations

$$
H_{\mathrm{fl}}=\sum_{q} E(q)\left(b^{+}(q) b(q)+\frac{1}{2}\right)
$$

where

$$
E(q)=2\left(\sqrt{\mathcal{K}_{\varphi \varphi}(q) \mathcal{K}_{z z}(q)}+\tilde{\mathcal{K}}_{z \varphi}(q)\right)
$$

(with $\left.\tilde{\mathcal{K}}_{z \varphi}(q)=\mathrm{i} \mathcal{K}_{z \varphi}(q)\right)$ is the spectrum of collective excitations.

It is instructive to compare the spectrum (19) with the Bogolyubov spectrum. Let us introduce formal notations $\epsilon, v, \gamma$ and $n$ and present Eq. (19) in the Bogolyubov form

$$
E(q)=\sqrt{\epsilon(\epsilon+2 \gamma n)}+\hbar q v
$$


In (20) the kinetic energy is defined as

$$
\epsilon=2 \mathcal{F}_{\mathrm{D}}(Q)-\mathcal{F}_{\mathrm{D}}(q+Q)-\mathcal{F}_{\mathrm{D}}(q-Q) .
$$

In the long wave limit $\left(q, Q \ll l^{-1}\right)$ this quantity is reduced to the standard expression for the kinetic energy $\epsilon=\hbar^{2} q^{2} / 2 M$, where

$$
M=\frac{2 \varepsilon \hbar^{2}}{e^{2} l}\left[\sqrt{\frac{\pi}{2}}\left(1+\frac{d^{2}}{l^{2}}\right) \exp \left(\frac{d^{2}}{2 l^{2}}\right) \operatorname{erfc}\left(\frac{d}{\sqrt{2} l}\right)-\frac{d}{l}\right]^{-1}
$$

is the magnetic mass of the pair (see, for instance, [22]).

In (20) the density is defined as $n=\nu(1-\nu) / 2 \pi l^{2}$. In the limit $\nu \rightarrow 0$ (or $(1-\nu) \rightarrow 0)$ this quantity coincides with the density of electron-hole pairs (in this case one can easily mark out the pairs from the background, see Figure 2). The factor $\nu(1-\nu)$ appears due to the electron-hole symmetry of the problem.

The superfluid velocity in (20) is defined as

$$
v=\frac{\mathcal{F}_{\mathrm{D}}(q+Q)-\mathcal{F}_{\mathrm{D}}(q-Q)}{\hbar q}(2 \nu-1) .
$$

At $q, Q \ll l^{-1}$ it reduces to the expression $v=(\hbar Q / M)(1-2 \nu)$ that differs from the common expression for the superfluid velocity by the factor $1-2 \nu$. At small $\nu$ this factor approaches unity and the difference disappears, but at zero imbalance $(\nu=1 / 2)$ it is equal to zero and the last term in (20) vanishes. This feature can be understood from the following arguments. The sign of $Q$ (gradient of the phase) determines the direction of the current. If one describes the supercurrent as motion of electron-hole pairs then the direction of the current depends on the direction of the superfluid velocity and on the direction of polarization of electron-hole pairs (Figure 2). Therefore at given $Q$ the filling factors $\nu<1 / 2$ and $\nu>1 / 2$ corresponds to opposite directions of superfluid velocities. The factor $(1-2 \nu)$ changes its sign under substitution $\nu \rightarrow 1-\nu$ and its appearance in the expression for $v$ reflects to the electron-hole symmetry of the problem.

The interaction parameter $\gamma$ in (20) is given by the expression

$$
\gamma=8 \pi l^{2}\left[\mathcal{H}(q)-\mathcal{F}_{\mathrm{S}}(q)+\frac{\mathcal{F}_{\mathrm{D}}(q+Q)+\mathcal{F}_{\mathrm{D}}(q-Q)}{2}\right] .
$$

The first term in (24) is caused by the direct Coulomb interaction between dipoles, two other terms correspond to the exchange interaction. One can see that at small $q$ the direct interaction term reduces to $\gamma_{0}=4 \pi e^{2} d / \varepsilon$. It is just the interaction parameter for a two-dimensional gas of classical dipoles (polarized perpendicular to the layer). We also note that in the limit $d \rightarrow 0$ (in which the pairs do not interact with each other) the quantity (24) approaches zero, and the collective excitation spectrum (20) turns to the spectrum of free particles $E(q)=\epsilon=\hbar^{2} q^{2} / 2 M$ (at $Q=0$ and $q l \ll 1$ ).

Thus, the spectrum (19) is similar to the Bogolyubov spectrum but there are certain differences caused by the electron-hole symmetry. The answer (19) at $\nu=1 / 2$ coincides with the spectrum obtained in [20]. In the low density limit $\nu \ll 1$ (low concentration of the pairs) and at small $q$ the result (19]) reduces to one found in [23] on the base of the Gross-Pitaevskii equation. 
According to the Landau criterium the energy (19) should be positive and realvalued for all $q$. At $d>d_{\mathrm{c}}$ (critical $d_{\mathrm{c}}$ depends on $\nu$ ) the spectrum becomes complexvalued already at $Q=0$. It means that at given $d$ and $\nu$ the superfluid state cannot be realized at all. At $d<d_{\mathrm{c}}$ the Landau condition violates at $Q>Q_{\mathrm{c}}$. The value $Q_{\mathrm{c}}$ (that depends on $\nu$ and $d / l$ ) determines the critical current. The relation between $Q_{\mathrm{c}}$ and the critical current can be obtained as follows. At nonzero vector potential the phase gradient $Q$ in the energy ([6]) should be replaced with the gauge invariant quantity

$$
Q \rightarrow Q-(e / \hbar c)\left(\mathbf{A}_{1}-\mathbf{A}_{2}\right)
$$

where $\mathbf{A}_{i}$ is a vector potential in the $i$-th layer. In a given layer the current (density of the current) is obtained from the equation $j_{n}=c \mathrm{~d} \epsilon / \mathrm{d} A_{n}$, where $\epsilon=E^{(0)} / S$ is the energy per unit area. Taking into account (25), one finds $j_{1}=-j_{2}=-(e / \hbar S) \mathrm{d} E^{(0)} / \mathrm{d} Q$, or, in the explicit form,

$$
j_{1}=-j_{2}=\frac{e}{\hbar} \frac{1}{4 \pi l^{2}} \sin ^{2} \theta_{0} \frac{\mathrm{d} \mathcal{F}_{\mathrm{D}}(Q)}{\mathrm{d} Q} .
$$

Substituting Eq.(8) into (26) one obtains the following relation between $Q$ and the current

$$
j_{1}=-j_{2}=-\frac{e^{3}}{2 \pi \varepsilon l^{2} \hbar} \nu(1-\nu) \int_{0}^{\infty} \mathrm{d} \tilde{k} \mathrm{e}^{-\frac{\tilde{k}^{2}}{2}} \tilde{k} J_{1}(\tilde{k} Q l) \mathrm{e}^{-\tilde{k} d / l} .
$$

At small $Q(Q \ll l)$ the current is proportional to the phase gradient: $j_{1}=(e / \hbar) \rho_{\mathrm{s}} Q$, where $\rho_{\mathrm{s}}=\hbar^{2} n / M$ is the superfluid stiffness.

One can easy check that the value of integral in (27) at any $Q$ and $d$ does not exceed 0.45 . Therefore, in any case the supercurrent is less than $j_{\mathrm{m}} \approx 0.018 e^{3} / \varepsilon l^{2} \hbar$. At typical parameters $\varepsilon=12.5, l=100 \AA$ one evaluates $j_{\mathrm{m}} \approx 5 \mathrm{~A} / \mathrm{m}$. Note this value of counterflow currents corresponds to very small value of parallel to the layers component of magnetic field produced by the current $\left(B_{\mathrm{y}} \approx 6 \cdot 10^{-6} \mathrm{~T}\right)$. Under accounting the Landau criterium this quantity is even smaller.

Now let us discuss the influence of tunnelling on the critical current. In general case the tunnelling reduces the critical current, moreover it may destroy the superfluid state completely [20]. To explain this effect it is instructive to introduce the Josephson length $\lambda=l \sqrt{2 \pi \rho_{s} / t \sqrt{\nu(1-\nu)}}$ (see, for instance, [19, 20]). This quantity determines the size of the soliton (Josephson vortex) in the bilayer quantum Hall system. If the Josephson length is small $\lambda<2 \pi / Q_{c}$ and the gradient of the phase caused by the soliton is large (larger than the critical gradient $Q_{c}$ ) the tunnelling influences significantly on the critical parameters. But such $\lambda$ correspond to quite large tunnelling amplitudes $\left(t / E_{C}>10^{-2}\right.$ for $\nu=1 / 2$ and $\left.d \approx l\right)$. Thus, in a common experimental situation $\left(t / E_{C} \sim 10^{-6}\right)$ the tunnelling can be neglected almost for all relevant values of $\nu$ and $d / l$. 


\section{The excitation spectrum and critical parameters for the four-layer system}

Let us turn to the consideration of the four-layer quantum Hall system where a twocomponent superfluid gas of electron-hole pairs can emerge. For definiteness, we consider the system with equal distances between the adjacent layers. We specify the case where supercurrents in both components are directed along the same axis.

The Hamiltonian of the system has the form (1) (with the summation over four layers). According to the result of Refs. [18] the electron-hole pairs are formed separately in the $n=1,2$ bilayer complex and in the $n=3,4$ bilayer complex. Correspondingly, the many-body wave function can be presented as a product of the functions (3):

$|\psi\rangle=\prod_{X}\left(\cos \frac{\theta_{\mathrm{T} X}}{2} c_{1 X}^{+}+\sin \frac{\theta_{\mathrm{T} X}}{2} \mathrm{e}^{\mathrm{i} \varphi_{\mathrm{T} X}} c_{2 X}^{+}\right)\left(\cos \frac{\theta_{\mathrm{B} X}}{2} c_{3 X}^{+}+\sin \frac{\theta_{\mathrm{B} X}}{2} \mathrm{e}^{\mathrm{i} \varphi_{\mathrm{B} X}} c_{4 X}^{+}\right)|0\rangle$.

In the state (28) the energy of the system consists of three terms:

$$
E=E_{\mathrm{T}}+E_{\mathrm{B}}+E_{\mathrm{TB}}
$$

In (29) $E_{\mathrm{T}}$ and $E_{\mathrm{B}}$ are the bilayer energies (given by Eq. (41)). The cross term has the form

$$
E_{\mathrm{TB}}=\frac{1}{2 L_{\mathrm{y}}} \sum_{X, X^{\prime}} H_{\mathrm{TB}}\left(X-X^{\prime}\right) \cos \theta_{\mathrm{T}}(X) \cos \theta_{\mathrm{B}}\left(X^{\prime}\right)
$$

where $H_{\mathrm{TB}}(X)=l^{2} \int_{-\infty}^{\infty} \mathrm{d} q \mathrm{e}^{\mathrm{i} q X} \mathcal{H}^{\mathrm{TB}}(q)$ with

$$
\mathcal{H}^{\mathrm{TB}}(q)=\frac{e^{2}}{2 \varepsilon l^{2}} \mathrm{e}^{-d|q|} \frac{\left(1-\mathrm{e}^{-d|q|}\right)^{2}}{|q|} \mathrm{e}^{-\frac{q^{2} l^{2}}{2}} .
$$

Note that in the state (28) the cross energy does not contain the exchange part.

The stationary homogeneous state is described by four parameters: $\nu_{i}=(1+$ $\left.\cos \theta_{0 i}\right) / 2$ and $Q_{i}=\mathrm{d} \varphi_{i X} / \mathrm{d} X(i=\mathrm{T}, \mathrm{B})$. The energy in this state reads as

$$
E^{(0)}=E_{\mathrm{T}}^{(0)}+E_{\mathrm{B}}^{(0)}+\frac{S}{4 \pi l^{2}} \mathcal{H}^{\mathrm{TB}}(0) \cos \theta_{0 \mathrm{~T}} \cos \theta_{0 \mathrm{~B}}
$$

where $E_{\mathrm{T}}^{(0)}\left(E_{\mathrm{B}}^{(0)}\right)$ are determined by the equation (6) with $\theta_{0}=\theta_{0 \mathrm{~T}}\left(\theta_{0 \mathrm{~B}}\right)$ and $Q=Q_{\mathrm{T}}\left(Q_{\mathrm{B}}\right)$.

The energy of fluctuations is found by the same procedure as for the bilayer system. The result is

$$
\begin{aligned}
& E_{\mathrm{fl}}=\frac{S}{4 \pi l^{2}} \sum_{q}\left\{\sum _ { i = \mathrm { T } , \mathrm { B } } \left[\tilde{m}_{i, z}(-q) \mathcal{K}_{z z}^{i i}(q) \tilde{m}_{i, z}(q)+2 \tilde{m}_{i, z}(-q) \mathcal{K}_{z \varphi}^{i i}(q) \tilde{\varphi}_{i}(q)\right.\right. \\
& \left.\left.+\tilde{\varphi}_{i}(-q) \mathcal{K}_{\varphi \varphi}^{i i}(q) \tilde{\varphi}_{i}(q)\right]+\tilde{m}_{\mathrm{T}, z}(-q) \mathcal{K}_{z z}^{\mathrm{TB}}(q) \tilde{m}_{\mathrm{B}, z}(q)\right\}
\end{aligned}
$$

where diagonal in $i$ components of the matrix $\mathcal{K}$ are given by the expressions (12)-(14), and the non-diagonal in $i$ component is $\mathcal{K}_{z z}^{\mathrm{TB}}(q)=\mathcal{H}^{\mathrm{TB}}(q)$. 
Replacing $\tilde{m}_{i, z}(q)$ and $\tilde{\varphi}_{i}(q)$ by the operators

$$
\begin{aligned}
& \hat{m}_{i, z}(q)=\sqrt{\frac{2 \pi l^{2}}{S}}\left(\frac{\mathcal{K}_{\varphi \varphi}^{i i}(q)}{\mathcal{K}_{z z}^{i i}(q)}\right)^{\frac{1}{4}}\left(b_{i, q}+b_{i,-q}^{+}\right), \\
& \hat{\varphi}_{i}(q)=\mathrm{i} \sqrt{\frac{2 \pi l^{2}}{S}}\left(\frac{\mathcal{K}_{z z}^{i i}(q)}{\mathcal{K}_{\varphi \varphi}^{i i}(q)}\right)^{\frac{1}{4}}\left(b_{i, q}-b_{i,-q}^{+}\right)
\end{aligned}
$$

we obtain the Hamiltonian written in terms of Bose creation and annihilation operators $H_{\mathrm{fl}}=\sum_{q}\left[\sum_{i=\mathrm{T}, \mathrm{B}}\left(E_{0, i}(q)+\hbar q v_{i}\right)\left(b_{i, q}^{+}(q) b_{i, q}+\frac{1}{2}\right)+g_{q}\left(b_{\mathrm{T}, q}^{+} b_{\mathrm{B}, q}+b_{\mathrm{T}, q} b_{\mathrm{B},-q}+h . c.\right)\right]$

with $E_{0, i}(q)=2 \sqrt{\mathcal{K}_{\varphi \varphi}^{i i}(q) \mathcal{K}_{z z}^{i i}(q)}$ and

$$
g_{q}=\frac{1}{2} \mathcal{H}^{\mathrm{TB}}(q) \sqrt[4]{\frac{\mathcal{K}_{\varphi \varphi}^{\mathrm{TT}}(q) \mathcal{K}_{\varphi \varphi}^{\mathrm{BB}}(q)}{\mathcal{K}_{z z}^{\mathrm{TT}}(q) \mathcal{K}_{z z}^{\mathrm{BB}}(q)}}
$$

In (35) the quantities $v_{i}$ are given by Eq. (23) (with $Q=Q_{i}$ and $\nu=\nu_{i}$ ).

The Hamiltonian (35) coincides in form with one obtained in [6] for the twocomponent superfluid Bose gas. Its diagonalization yields

$$
E=\sum_{q} \sum_{\alpha=1,2} E_{\alpha}(q)\left(b_{\alpha, q}^{+} b_{\alpha, q}+\frac{1}{2}\right)
$$

where $b_{\alpha}^{+}\left(b_{\alpha}\right)$ are the operators of creation (annihilation) of collective excitations, $E_{\alpha}(q)$ are the excitation spectra. The dispersion equation for the spectra is analogous to one obtained [10]:

$\left[E_{0, \mathrm{~T}}^{2}(q)-\left(E-\hbar v_{\mathrm{T}} q\right)^{2}\right]\left[E_{0, \mathrm{~B}}^{2}(q)-\left(E-\hbar v_{\mathrm{B}} q\right)^{2}\right]-4 g_{q}^{2} E_{0, \mathrm{~T}}(q) E_{0, \mathrm{~B}}(q)=0$.

One can show (see [10]) that the energies of collective excitations are real valued and positive if the quantities $E_{0, j}(q)$ are real valued for all $q$, and the following inequalities are satisfied

$$
\begin{aligned}
& {\left[E_{0, \mathrm{~T}}^{2}(q)-\left(\hbar v_{\mathrm{T}} q\right)^{2}\right]\left[E_{0, \mathrm{~B}}^{2}(q)-\left(\hbar v_{\mathrm{B}} q\right)^{2}\right]-4 g_{q}^{2} E_{0, \mathrm{~T}}(q) E_{0, \mathrm{~B}}(q)>0,} \\
& E_{0, \mathrm{~T}}(q)-\hbar v_{\mathrm{T}}|q|>0
\end{aligned}
$$

(the condition (40) can be replaced by $\left.E_{0, \mathrm{~B}}(q)>\hbar v_{\mathrm{B}} q\right)$.

Putting $Q_{\mathrm{T}}=Q_{\mathrm{B}}=0$ and solving Eq. (38) one obtains the spectra of collective modes at zero currents. The requirement of real valued spectra yields the critical interlayer distance $d_{\mathrm{c}}$. The dependence of $d_{\mathrm{c}}$ on the filling factors is shown in Figure 3 (for $\nu_{\mathrm{T}}=\nu_{\mathrm{B}}$ ). The behavior of the critical interlayer distance is the same as for the bilayer system [24], but absolute values of $d_{\mathrm{c}}$ are a little bit smaller. The minimal critical distance $\left(d_{\mathrm{c} \text {,min }} \approx 1.015 \mathrm{l}\right)$ corresponds to the case of zero imbalance of filling factors. At $d>d_{\mathrm{c}, \mathrm{min}}$ one can say also about critical filling factors (that decreases under increase of $d)$.

A state with nonzero supercurrents can be realized only $d<d_{\mathrm{c}}\left(\nu_{\mathrm{T}}, \nu_{\mathrm{B}}\right)$ if for given $Q_{i}$ the spectra satisfy the inequalities (39), (40). The currents are determined by the relation $j_{i}=-(e / \hbar S) \mathrm{d} E^{(0)} / \mathrm{d} Q_{i}$. Since the cross term in Eq.(32) does not depend on 
$Q_{j}$, the relation between $Q_{i}$ and $j_{i}$ is same (Eq. (26)) as for the bilayer system (if one neglects the fluctuating part of energy, see Sec. 44). The inequalities (39), (40) determine a joint condition on $Q_{i}$ and the critical current of one component depends on the current of the other component.

The relations between the critical currents for $d / l=0.9$ and $\nu_{\mathrm{T}}=\nu_{\mathrm{B}}$ are shown in Figure 4. According to Figure 4, typical absolute values of the critical currents are less or of order of $1 \mathrm{~A} / \mathrm{m}$. Comparing the results presented in Figure 4 with ones of Ref. [10] one can see that the critical currents demonstrate the behavior similar to one of critical velocities in two-component superfluid Bose gases. Namely, the maximum supercurrent of one component can be reached at zero supercurrent of the other component, while at equal currents their allowed values are the smallest one. Since measurements of electrical currents in the layers are more simple than the measurements of superfluid velocities in two-component mixtures, quantum Hall four-layer systems can be used for the observation of specific behavior of critical velocities in two-components superfluids [10].

\section{Non-dissipative drag between the components}

Eq.(26) used in the previous section for the calculation of the current does not take into account the energy of fluctuations. Therefore, the results obtained are valid at temperatures much smaller than the Coulomb energy. In two-component systems the fluctuations yield an additional contribution to the current even at zero temperatures. This contribution is caused by the energy of zero-point oscillations. It is rather small contribution and it can be neglected under calculations of the critical currents. Nevertheless, this contribution determines a new effect - a non-dissipative drag between the components. The value of the non-dissipative drag decreases under increase of the temperature, but the decrease of the drag factor is essential at temperatures larger than the interaction energy [6]. Here, for simplicity, we consider the case of zero temperatures and small phase gradients $\left(Q_{i} l \ll 1\right)$.

Taking into account the zero-point oscillations energy

$$
E=E^{(0)}+\frac{1}{2} \sum_{\alpha=1,2} \sum_{\mathbf{q}} E_{\alpha}(\mathbf{q})
$$

and expanding it in series in $Q_{i}$ one obtains the following expression for the energy

$$
E \approx E_{0}+\frac{S}{2} \sum_{i k} \Lambda_{i k} Q_{i} Q_{k},
$$

where $E_{0}$ is independent of $Q_{i}$, and $\boldsymbol{\Lambda}$ is some symmetric real matrix. The mean-field energy $E^{(0)}$ in (41) is diagonal in $Q_{i}$, but the zero-point oscillation energy contains a non-diagonal term. Due to this a supercurrent of a given component depends on the phase gradients of both components

$$
j_{i}=\frac{e}{\hbar} \sum_{k} \Lambda_{i k} Q_{k} \text {. }
$$


The latter results in the non-dissipative drag effect. Indeed, let the current in the drive component (e.g. component $\mathrm{T}$ ) is given (it is fixed by an external source) while the current in the drag component $(\mathrm{B})$ is not fixed. The value of current in the drag component can be found from the requirement of minimum of the energy Eq. (42) subjected to the constrain $j_{\mathrm{T}}=$ const. One can see that the drag current is nonzero and proportional to the non-diagonal component of the matrix $\Lambda$ :

$$
j_{\mathrm{B}}=\frac{\Lambda_{\mathrm{BT}}}{\Lambda_{\mathrm{TT}}} j_{\mathrm{T}}
$$

We define the drag factor as the ratio of the drag current to the drive current: $f_{\mathrm{dr}}=\Lambda_{\mathrm{BT}} / \Lambda_{\mathrm{TT}}$. As was found in [5, 6] the drag factor for the atomic Bose gases is rather small - the most optimistic estimates yield $f_{\mathrm{dr}} \sim 10^{-4}$. Let us compute the drag factor for the quantum Hall multilayers.

The main contribution into the diagonal components of $\boldsymbol{\Lambda}$ comes from the energy $E^{(0)}$, and the quantity $\Lambda_{\mathrm{TT}}$ in the leading order is evaluated as $\Lambda_{\mathrm{TT}}=\hbar^{2} n_{\mathrm{T}} / M$ $\left(n_{i}=\nu_{\mathrm{i}}\left(1-\nu_{\mathrm{i}}\right) / 2 \pi l^{2}\right)$. The non-diagonal component of $\boldsymbol{\Lambda}$ is caused by the zero-point oscillation energy. Strictly spearing, to compute this energy one should obtain the spectrum of collective excitations for all $\mathbf{q}$ (not only for $\mathbf{q} \| \hat{x}$ ). But if one needs only non-diagonal in $Q_{i}$ term and $Q_{i}$ are assumed to be small an approximate dispersion equation can be used. This equation is obtained from (38) if one replaces the quantities $\hbar v_{i} q$ with $\hbar v_{i} q_{x}$ and neglects the dependencies of $E_{0, i}$ on $Q_{i}$. This approximation can be justified under accounting that in the series for $E_{\alpha}(\mathbf{q})$ the $Q_{T} Q_{B}$ terms comes only from the product $v_{\mathrm{B}} v_{\mathrm{T}}$ (see [6]).

Such an approximation yields the dispersion equation

$$
\left[E_{\mathrm{T}}^{2}-\left(E-\hbar v_{\mathrm{T}} q_{x}\right)^{2}\right]\left[E_{\mathrm{B}}^{2}-\left(E-\hbar v_{\mathrm{B}} q_{x}\right)^{2}\right]-4\left(\gamma^{\prime}\right)^{2} \epsilon^{2} n_{\mathrm{T}} n_{\mathrm{B}}=0
$$

where $E_{i}=\sqrt{\epsilon\left[\epsilon+2 \gamma n_{i}\right]}$ are the spectra of excitations for decoupled one-component systems, $\epsilon=2\left[\mathcal{F}_{\mathrm{D}}(0)-\mathcal{F}_{\mathrm{D}}(q)\right]$ is the kinetic energy of electron-hole pairs, $\gamma=$ $8 \pi l^{2}\left[\mathcal{H}(q)-\mathcal{F}_{\mathrm{S}}(q)+\mathcal{F}_{\mathrm{D}}(0)\right]$ and $\gamma^{\prime}=4 \pi l^{2} \mathcal{H}^{\mathrm{TB}}(q)$ are the Fourier components of the interaction potentials, and $v_{i}=(2 / \hbar q)\left(\mathrm{d} \mathcal{F}_{\mathrm{D}}(q) / \mathrm{d} q\right)\left(2 \nu_{i}-1\right) Q_{i}$ are the superfluid velocities (with the factors that account the electron-hole symmetry).

Eq. (45) coincides in form with one for the atomic two-component Bose gases [6, 10]. For obtaining $\Lambda_{\mathrm{BT}}$ we present the solutions of (45) as series in $v_{i}$ and substitute them into (41). The details of such a procedure are described in [6]. Here we present the final expression for the drag factor

$$
f_{\mathrm{dr}}^{\mathrm{BT}}=\frac{2 M}{\pi \hbar^{2} n_{\mathrm{T}}}\left(1-2 \nu_{\mathrm{T}}\right)\left(1-2 \nu_{\mathrm{B}}\right) \int_{0}^{\infty} \frac{\left(\gamma^{\prime}\right)^{2} n_{\mathrm{T}} n_{\mathrm{B}} \epsilon^{2}}{E_{\alpha} E_{\beta}\left(E_{\alpha}+E_{\beta}\right)^{3}}\left(\frac{\mathrm{d} \mathcal{F}_{\mathrm{D}}}{\mathrm{d} q}\right)^{2} q \mathrm{~d} q,(
$$

where

$$
E_{\alpha(\beta)}=\sqrt{\frac{E_{\mathrm{T}}^{2}+E_{\mathrm{B}}^{2}}{2} \pm \sqrt{\frac{\left(E_{\mathrm{T}}^{2}-E_{\mathrm{B}}^{2}\right)^{2}}{4}+4\left(\gamma^{\prime}\right)^{2} \epsilon^{2} n_{\mathrm{T}} n_{\mathrm{B}}}}
$$

are the energies of collective excitations at $Q_{\mathrm{T}}=Q_{\mathrm{B}}=0$. 
Since the problem has one energy parameter (the Coulomb energy $e^{2} / \varepsilon l$ ) the drag factor (46) depends only on dimensionless quantities $d / l, \nu_{\mathrm{T}}$ and $\nu_{\mathrm{B}}$. The dependence of the drag factor on the filling factor $\left(\nu=\nu_{\mathrm{T}}=\nu_{\mathrm{B}}\right)$ at different $d / l$ is shown in Figure 5. One can see that this dependence has an extremum at small $\nu$ (small density of the pairs). Similar feature was obtained for the atomic Bose gases with dipole-dipole interaction [5].

The specific feature is a sharp increase of the drag factor near critical $\nu$ (or $d$, see Figure 6). The effect is caused by a roton-like minimum in the spectrum of the lowest collective mode (Figure 7).

Other specific features are the vanishing of the drag effect at $\nu_{\mathrm{B}}=1 / 2\left(\nu_{\mathrm{T}}=1 / 2\right)$, and the alternation of the sign of the drag factor under change of sign of the filling factor imbalance $\left(\nu_{\mathrm{T}}\right.$ to $1-\nu_{\mathrm{T}}$ or $\nu_{\mathrm{B}}$ to $\left.1-\nu_{\mathrm{B}}\right)$. The alternation of the direction of the drag current can be observed if one keeps one of the filling factor constant, while the other filling factor tunes from $\nu_{i}<1 / 2$ to $\nu_{i}>1 / 2$. This feature can be understood from the electron-hole symmetry argumentation (see the discussion in Sec. 2).

But the main feature is large absolute values of the drag factor (in $10^{2} \div 10^{3}$ times larger than the most optimistic figures for the atomic Bose gases). Large values of the effect are caused by a number of factors. As follows from the consideration [5, 6] the drag effect in two-dimensional systems can be larger than in three-dimensional ones, but for Bose gases in bilayer traps large values are not reached due to weak interspecie interaction. Fortunately, in quantum Hall multilayers the interaction between different superfluid components is of the same order as the interaction inside a given component. Moreover, the intra-component interaction is reduced due to the exchange interaction. At last, the drag effect is enhanced considerable by the presence of the roton-like minimum in the energy spectrum, and this minimum becomes deeper at interlayer distances or filling factors close to critical ones (Figure 7). All these factors work in favor of the drag effect and result in a giant drag factor in comparison with atomic two-component Bose systems.

\section{Conclusion}

In conclusion, we have studied superfluid properties of a two-component gas of electronhole pairs in a quantum Hall four-layer system. We have found that the critical parameters (critical interlayer distance and critical currents) for this system are slightly less but of the same order as in bilayers. The critical currents in the two-component gas of electron-hole pairs demonstrate the behavior similar to one of atomic two-component Bose gases. In particular, the largest value of the critical current in a given component can be reached if the current in the other component is equal to zero. In multilayer quantum Hall systems this peculiar behavior of two-components superfluids can be observed by electrical measurements. The non-dissipative drag effect between the components is predicted. The effect takes place only at nonzero imbalance of filling factors of each (top and bottom) bilayer, and the drag current alternates its direction 
under change of sign of the imbalance in one of the bilayers. The drag factors is quite large, and the largest values can be achieved at the interlayer distances close to the critical ones.

\section{References}

[1] Andreev A F and Bashkin E P 1975 Zh. Exp. Teor. Fiz. 69319 [1975 Sov. Phys. JETP 42 164].

[2] Duan J-M and Yip S 1993 Phys. Rev. Lett. 703647.

[3] Tanatar B, Das A K 1996 Phys. Rev. B 5413827.

[4] Terentjev S V and Shevchenko S I 1999 Fiz. Nizk. Temp. 25664 [ 1999 Low Temp. Phys. 25 493].

[5] Fil D V and Shevchenko S I 2004 Fiz. Nizk. Temp. 301028 [2004 Low Temp. Phys. 30 770].

[6] Fil D V and Shevchenko S I 2005 Phys. Rev. A 72013616.

[7] Rojo A 1999 J. Phys.: Cond. Matt. 11 R31.

[8] Alpar M A, Langer S A and Sauls J A 1984 Astrophys. J. 282533.

[9] Babaev E 2004 Phys.Rev. D 70043001.

[10] Kravchenko L Yu and Fil D V 2008 J. Low Temp. Phys. 150162.

[11] Hall D S, Matthews M R, Ensher J R, Wieman C E and Cornell E A 1998 Phys. Rev. Lett. 81 1539 .

Maddaloni P, Modugno M, Fort C, Minardi F and Inguscio M 2000 Phys. Rev. Lett. 852413.

Miesner H-J, Stamper-Kurn D M, Stenger J, Inouye S, Chikkatur A P and Ketterle W 1999 Phys. Rev. Lett. 822228.

Modugno G, Modugno M, Riboli F, Roati G and Inguscio M 2002 Phys. Rew. Lett. 89190404.

Mudrich M, Kraft S, Grimm R, Mosk A and Weidenmüller M 2002 Phys. Rev. Lett. 88253001.

[12] MacDonald A H and Rezayi E H 1990 Phys. Rev. B 423224.

Wen X-G and Zee A 1992 Phys. Rev. Lett. 691811.

Moon K, Mori H, Yang K, Girvin S M, MacDonald A H, Zheng L, Yoshioka D and Zhang S-C 1995 Phys. Rev. B. 515138.

[13] Spielman I B, Eisenstein J P, Pfeiffer L N and West K W 2000 Phys. Rev. Lett. 845808.

Spielman I B, Eisenstein J P, Pfeiffer L N and West K W 2001 Phys. Rev. Lett. 87036803.

Kellogg M, Spielman I B, Eisenstein J P, Pfeiffer L N and West K W 2002 Phys. Rev. Lett. 88 126804 .

Kellogg M, Eisenstein J P, Pfeiffer L N and West K W 2003 Phys. Rev. Lett. 90246801.

Spielman I B, Kellogg M, Eisenstein J P, Pfeiffer L N and West K W 2004 Phys. Rev. B 70081303.

Kellogg M, Eisenstein J P, Pfeiffer L N and West K W 2004 Phys. Rev. Lett. 93036801.

Eisenstein J P and MacDonald A H 2004 Nature (London) 432691.

[14] Tutuc E, Shayegan M and Huse D A 2004 Phys. Rev. Lett. 93036802.

[15] Wiersma R D, Lok J G S, Kraus S, Dietsche W, von Klitzing K, Schuh D, Bichler M, Tranitz H-P and Wegscheider W 2004 Phys. Rev. Lett. 93266805.

[16] Butov L V and Filin A I 1998 Phys. Rev. B. 581980.

Butov L V, Ivanov A L, Imamoglu A, Littlewood P B, Shashkin A A, Dolgopolov V T, Campman K L and Gossard A C 2001 Phys. Rev. Lett. 865608.

Butov L V, Lai C W, Ivanov A I, Gossard A C and Chemia D S 2002 Nature 41747.

[17] Larionov A V, Timofeev V B, Hvam J and Soerensen K 2002 Pisma Zh. Eksp. Teor. Fiz. 75233 [2002 JETP Letters 75 200].

Dremin A A, Timofeev V B, Larionov A V, Hvam J and Soerensen K 2002 Pisma Zh. Eksp. Teor. Fiz. 76526 [2002 JETP Letters 76 450].

[18] Shevchenko S I, Fil D V and Yakovleva A A 2004 Fiz. Nizk. Temp. 30431 [2004 Low Temp. Phys. 30 321].

Fil D V and Shevchenko S I 2005 Journal of Luminescence 112212.

[19] Fil D V and Shevchenko S I 2007 Fiz. Nizk. Temp. 331023 [2007 Low Temp. Phys. 33 780]. 
[20] Abolfath M, MacDonald A H and Radzihovsky L 2003 Phys. Rev. B 68155318.

[21] Simon S B 2005 Sol. St. Commun. 13481.

[22] Lozovok Yu E and Ruvinsky A M 1997 Zh. Eksp. Teor. Fiz. 1121791 [1997 JETP 85 979].

[23] Bezuglyj A I and Shevchenko S I 2007 Phys. Rev. B 75075322.

[24] Zhang C-H and Joglekar Y N Preprint arXiv:0711.4847. 


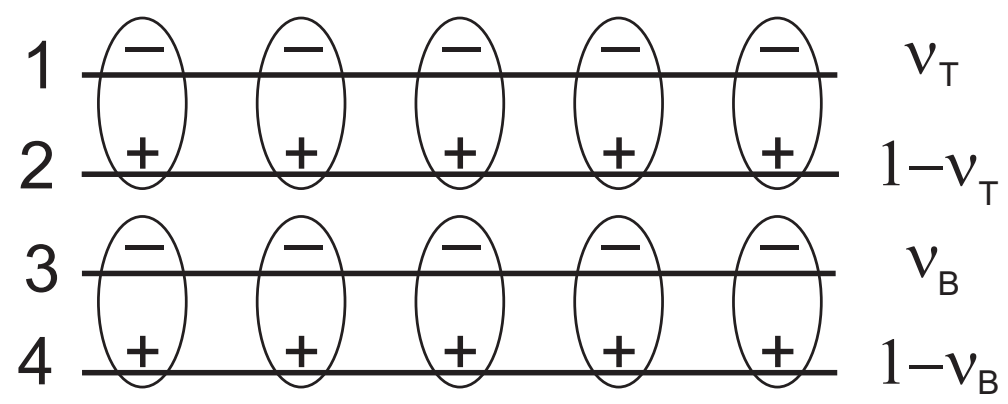

Figure 1. The four-layer system with two-component gas of electron-hole pairs.

a)

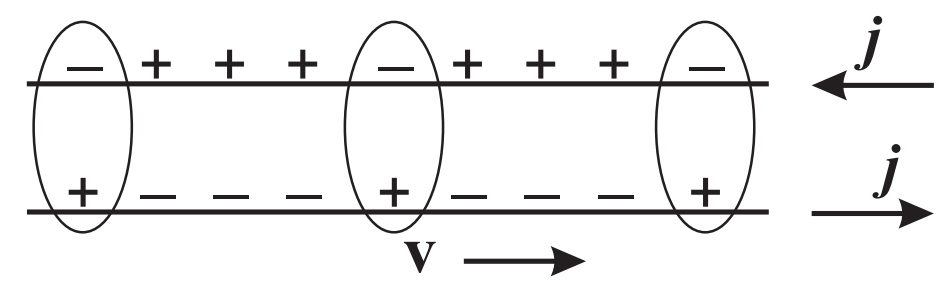

b)

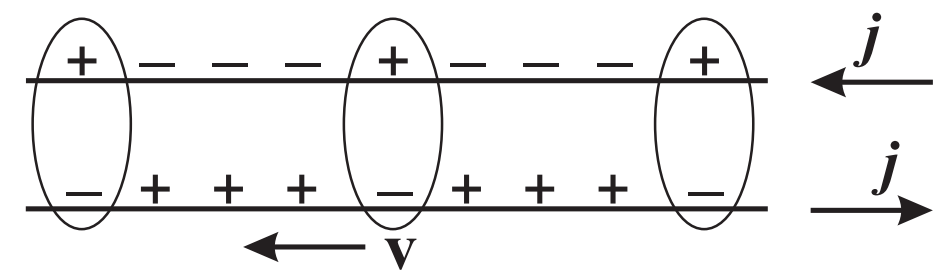

Figure 2. Relative directions of electrical currents in the layers $(j)$ and the velocities of electron-hole pairs (v) at $\nu<1 / 2$ (a) and $\nu>1 / 2(\mathrm{~b})$.

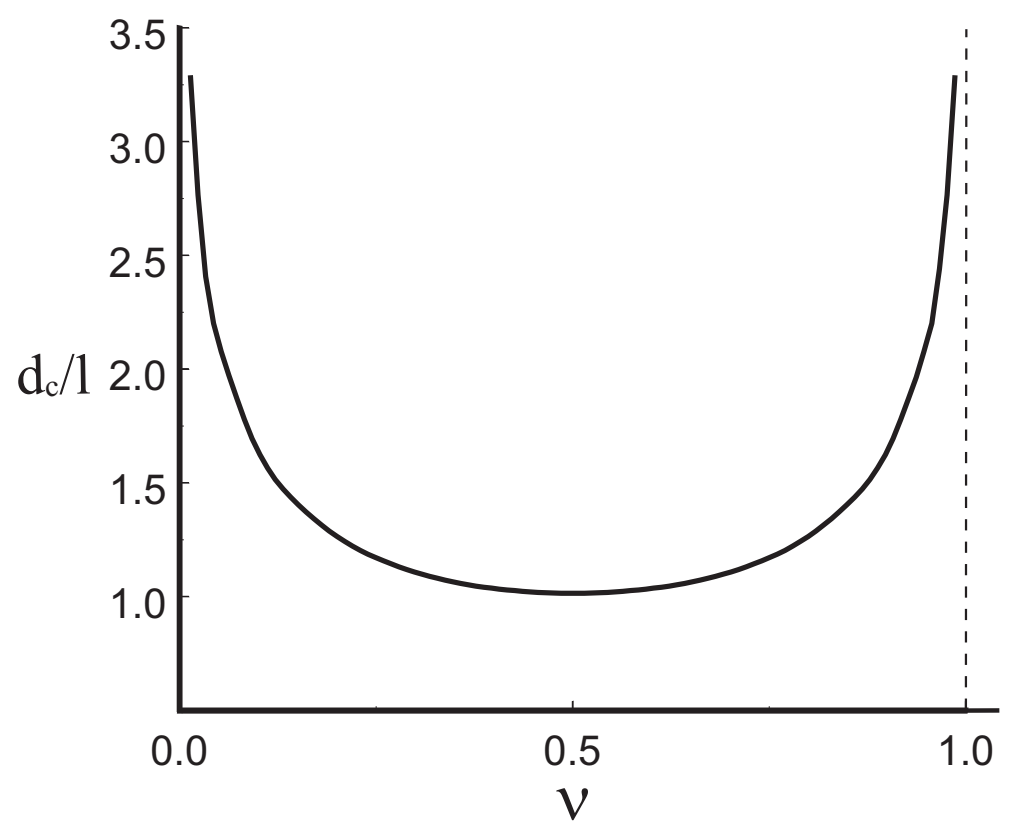

Figure 3. Critical interlayer distance for the four-layer system vs the filling factor $\left(\nu=\nu_{T}=\nu_{B}\right)$. 


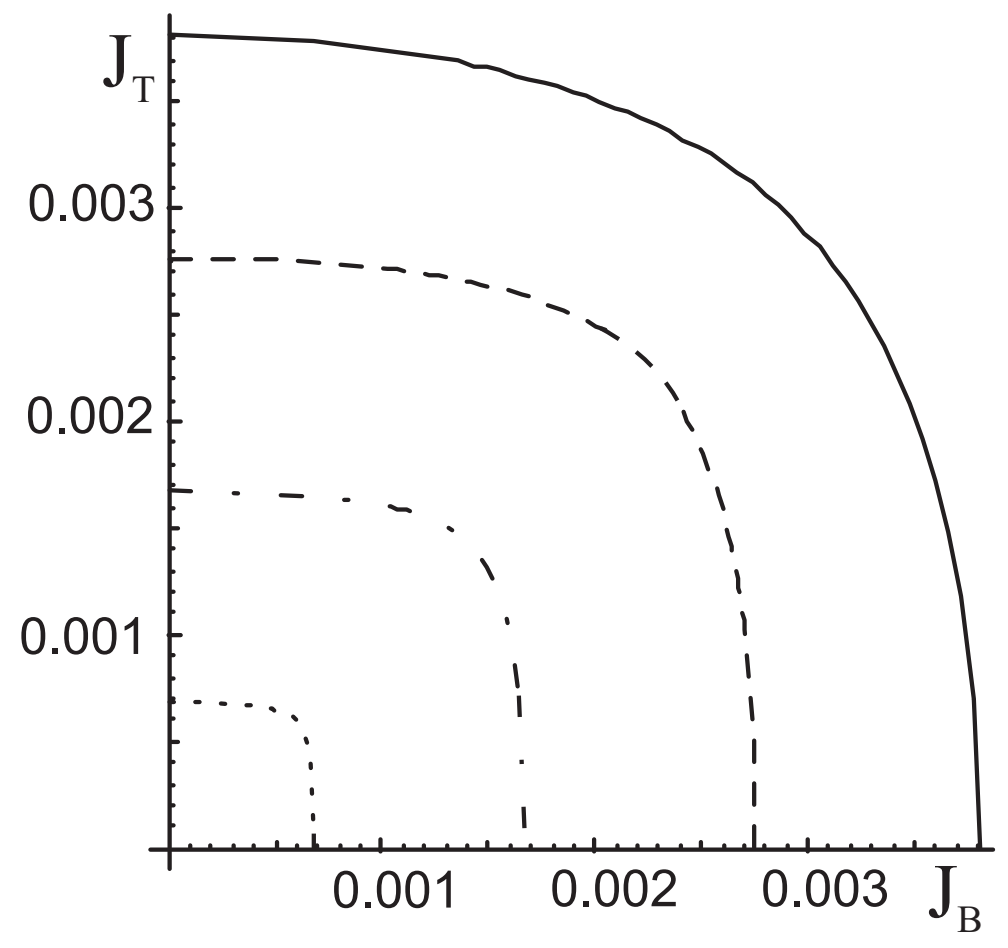

Figure 4. Critical currents (in $e^{3} / \hbar \varepsilon l^{2}$ units) at $d / l=0.9$ and the filling factors $\nu_{T}=\nu_{B}=0.5,0.25,0.15,0.07$ (from the top to the bottom curve)

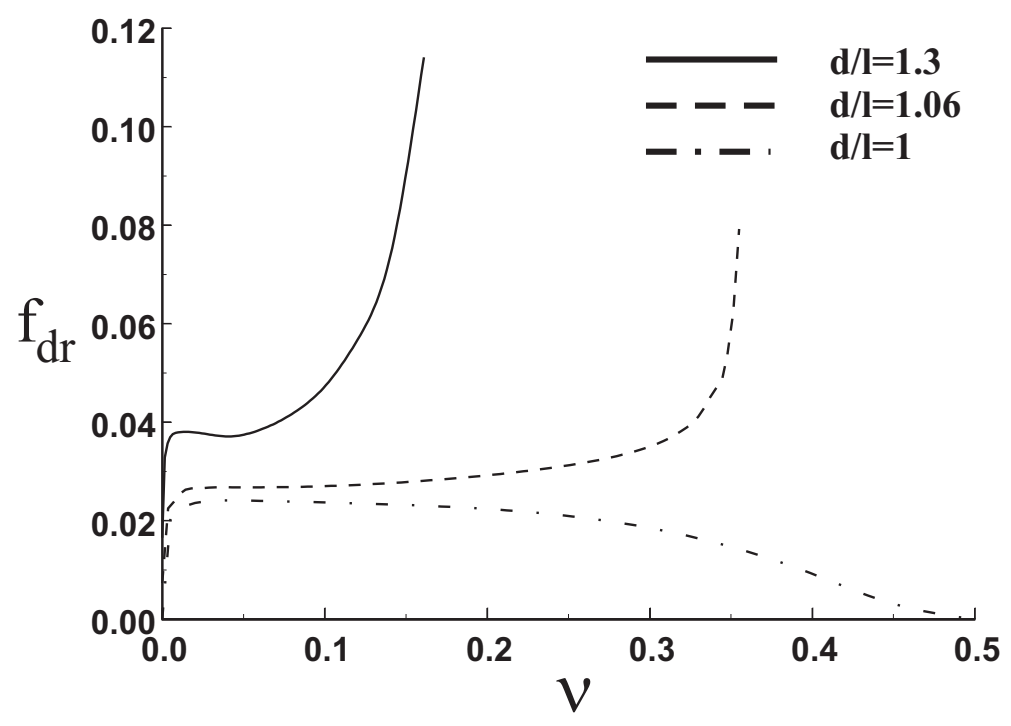

Figure 5. Drag factor vs the filling factor $\left(\nu=\nu_{T}=\nu_{B}\right)$. 


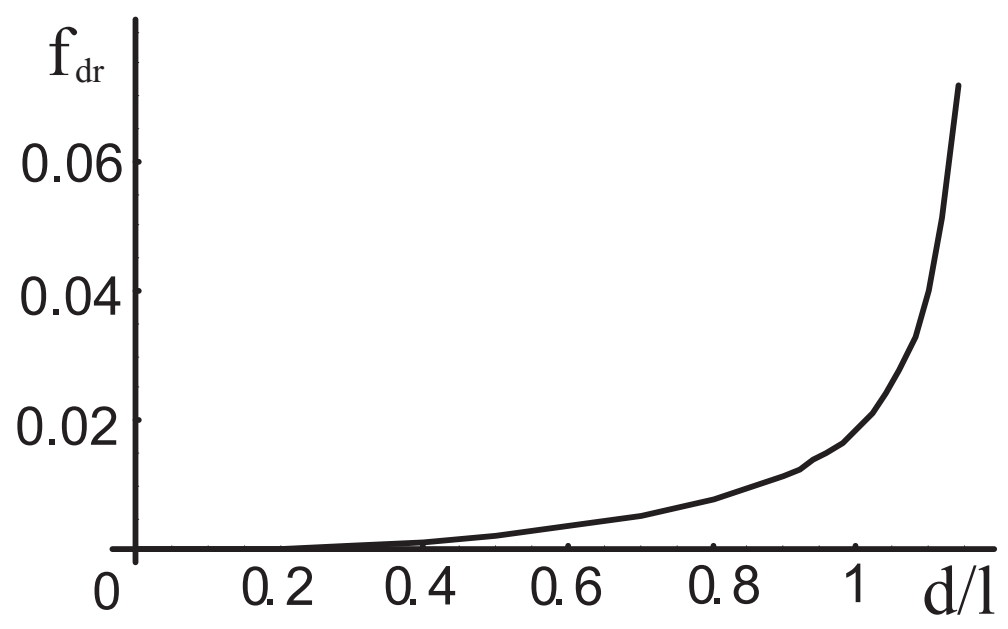

Figure 6. Drag factor vs the interlayer distance (at $\nu_{T}=\nu_{B}=1 / 4$ ).

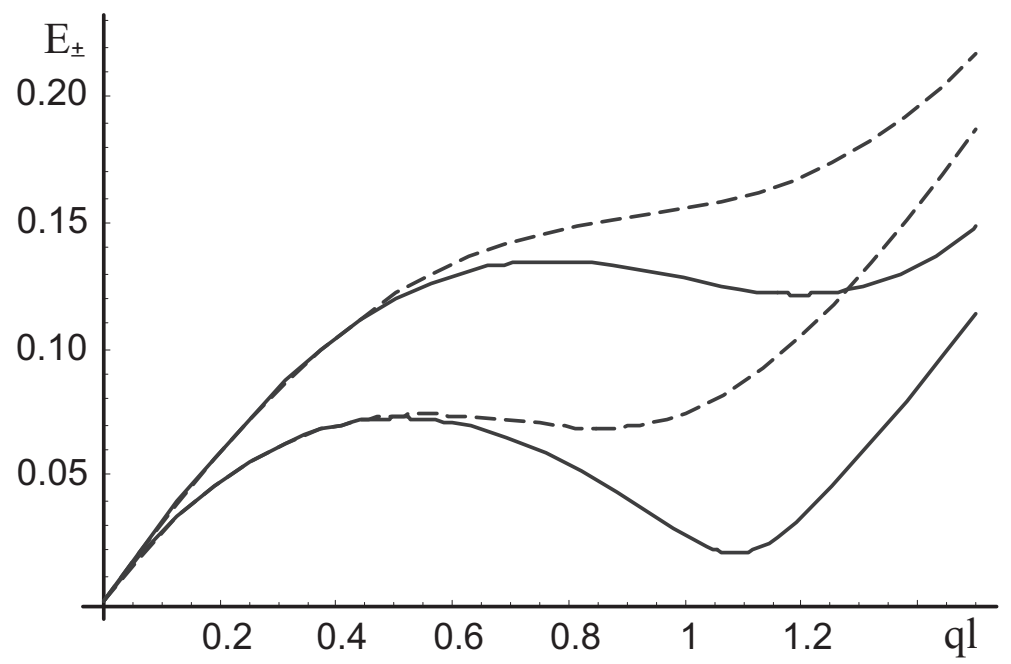

Figure 7. The energies (in $e^{2} / \varepsilon l$ units) of the collective modes at $\nu_{T}=\nu_{B}=1 / 4$. Dashed curves - $d=l$, solid curves - $d=1.16 l$ 